\section{Hipatia Press}

www.hipatiapress.com

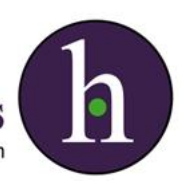

Instructions for authors, subscriptions and further details:

http://rise.hipatiapress.com

\title{
El Informe Coleman a Debate en su Cincuenta Aniversario
}

Alberto Álvarez-Sotomayor ${ }^{1}$

Gloria Martínez-Cousinou ${ }^{2}$

1) Universidad de Córdoba, Spain

2) Universidad Loyola Andalucía, Spain

Date of publication: June $25^{\text {th }}, 2016$

Edition period: June 2016-October 2016

To cite this article: Álvarez-Sotomayor, A., Martínez-Cousinou, G. (2016). El Informe Coleman a Debate en su Cincuenta Aniversario. International Journal of Sociology of Education, 5(2), 87-106. doi:

10.17583/rise.2016.2104

To link this article: http://dx.doi.org/10.17583/rise.2016.2104

\section{PLEASE SCROLL DOWN FOR ARTICLE}

The terms and conditions of use are related to the Open Journal System and to Creative Commons Attribution License (CC-BY) 



\section{Reassessing the Coleman Report on its 50th Anniversary}

\author{
Alberto Álvarez-Sotomayor \\ Universidad de Córdoba
}

Gloria Martínez-Cousinou

Universidad Loyola Andalucía

(Received: 17 May 2016; Accepted: 25 May 2016; Published: 25 June 2016)

\section{Abstract}

July 2016 will mark 50 years since the well-known Coleman Report was published. This report is undoubtedly a key publication in the field of Sociology of Education despite being considered extremely controversial upon its publication. This monographic issue of the International Journal of Sociology of Education has declared this anniversary as an opportunity to reassess some of the discussions generated from its main results. In this introductory paper, both the Coleman Report itself and subsequent debates over it are contextualized. An exploration of the social and political framework giving rise to the Report as well as its objectives, methodology and main issues are firstly analyzed. Additionally, its academic impact over the last five decades is also addressed. Finally, the main social and academic debate aroused by the Coleman Report is considered: the prominence of students' socioeconomic origin versus schooling factors when explaining education inequality. This debate is also the common thread which links the three subsequent articles included in this monographic issue.

Keywords: Coleman Report, academic achievement, socioeconomic status, school effects. 


\section{El Informe Coleman a Debate en su Cincuenta Aniversario}

\author{
Alberto Álvarez-Sotomayor \\ Universidad de Córdoba
}

Gloria Martínez-Cousinou

Universidad Loyola Andalucía

(Recibido: 17 de Mayo 2016; Aceptado: 25 de Mayo 2016; Publicado: 25 Junio 2016)

\section{Resumen}

En julio de 2016 se cumplen cincuenta años desde la publicación del conocido como Informe Coleman, sin duda, una de las publicaciones de mayor impacto para la sociología de la educación y que estuvo rodeada de una fuerte controversia desde un principio. Este número monográfico de la Revista Internacional de Sociología de la Educación aprovecha dicho aniversario para retomar algunos de los debates generados a partir de sus resultados. Con este primer artículo introductorio se pretende contextualizar esos debates y el propio informe. Para ello se recuerda el marco social y político que dio lugar al estudio de Coleman y sus colaboradores, así como sus objetivos, metodología y las principales cuestiones en él analizadas. Se aborda también el impacto que ha tenido a lo largo de estos cinco decenios. Por último, se sitúa al lector en el principal debate académico y social que suscitó el informe, aquel que gira en torno a la importancia que tiene sobre las desigualdades educativas el origen socioeconómico frente a los factores escolares. Este constituye también el eje principal de la discusión de los otros tres artículos que componen el número.

Palabras clave: Informe Coleman, rendimiento académico, desigualdades educativas, estatus socioeconómico, efectos de las escuelas 



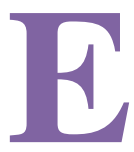

n julio de 1964 se aprueba en Estados Unidos la Civil Rights Act, una ley histórica en materia de derechos civiles que prohibía la discriminación basada en la raza, la religión, el sexo o el origen nacional de las personas. En su sección 402 establecía lo siguiente:

SEC. 402. The Commissioner [refiriéndose al Commissioner de Educación] shall conduct a survey and make a report to the President and the Congress, within two years of the enactment of this title, concerning the lack of availability of equal educational opportunities for individuals by reason of race, color, religion, or national origin in public educational institutions at all levels in the United States, its territories and possessions, and the District of Columbia. (Civil Rights Act, 1964).

Por lo tanto, se imperaba a la Oficina de Educación de los EE.UU. a que, en el plazo de dos años, llevara a cabo un estudio sobre la desigualdad de oportunidades educativas por razón de raza, religión u origen nacional. Unos meses más tarde, en febrero de 1965, James S. Coleman, por entonces profesor de Sociología en la Universidad John Hopkins, recibe una llamada telefónica del entonces assistant commissioner para las estadísticas de la Oficina de Educación ofreciéndole dirigir dicho estudio (Coleman, 1990, p.xi). En ese momento comienza la andadura de la investigación que daría lugar al Equality of Educational Opportunity Report (EEOR en lo sucesivo), más conocido como Informe Coleman, un trabajo de innegable impacto académico, político y social. Un trabajo que generó una controversia y un debate que, como ilustra el contenido de este monográfico, sigue acompañándole cincuenta años después. Pero volvamos al contexto en el que nace este estudio, porque ello nos ayudará a entender mejor su origen, así como parte de la controversia generada en su momento.

En la primera mitad de los años sesenta el movimiento por los derechos civiles de la población negra ${ }^{1}$ en EE.UU. se encontraba en un momento álgido. Se enfrentaba a una fuerte segregación racial que tenía su origen histórico en los inicios del periodo esclavista. Una segregación que todavía por entonces no era solo de facto, sino que tenía componentes formales (de jure) en la mayoría de los estados sureños. En ellos, por ejemplo, aún se 


\section{0 Álvarez-Sotomayor \& Martínez-Cousinou-Informe Coleman}

excluía a la población afroamericana del sistema político y electoral al negárseles el derecho al voto por medio de diversas restricciones legales que les afectaban directa y mayoritariamente.

Si hasta 1955 la lucha de este movimiento se había centrado en la acción judicial, a partir de ese año la estrategia cambió hacia la "acción directa". Los boicots, las sentadas, las marchas, las revueltas y los actos de desobediencia civil eran continuos y se extendían por todo el país, si bien tenían en el Sur su mayor foco. Fue, por ejemplo, en 1963 cuando tuvo lugar la famosa y multitudinaria marcha de Washington DC en la que Martin Luther King Jr. pronunció su célebre discurso ( $I$ have a dream). Curiosamente, el propio Coleman formó parte de uno de esos — por entonces habituales - actos reivindicativos. Fue un año y medio antes de recibir aquella llamada y un año antes de la aprobación de la Civil Rights Act. Él y su mujer acabaron detenidos tras intentar entrar con una familia negra a un parque en el que el acceso estaba permitido solo a personas blancas (Kilgore, 2016).

El movimiento por los derechos civiles de la población negra era, en gran medida, un movimiento anti-segregacionista y en él, la lucha contra la segregación racial en las escuelas estuvo en el centro de las reivindicaciones desde primera hora. De hecho, el conocido como caso de Brown contra el Consejo de Educación de Topeka, en Kansas, 1954, en el cual el Tribunal Supremo de los EE.UU. declaró inconstitucionales las leyes estatales que permitían a las escuelas públicas separar al alumnado negro y al blanco, es considerado un acontecimiento histórico dentro del movimiento. Como lo fue también la crisis de "los nueve de Little Rock", en Arkansas. Esta se originó tras el rechazo del Gobernador de este y otros estados sureños a aplicar la resolución del conocido como caso Brown II, el cual ordenaba a que esta forma de segregación fuera eliminada "con toda la celeridad posible". ${ }^{2}$

Otro elemento clave para entender el origen y el impacto del EEOR es el contexto político del momento. La investigación llevada a cabo por Coleman y sus colaboradores coincidió con la administración presidida por Lyndon B. Johnson y con las medidas adoptadas por esta en el marco de su Great Society. La "Gran Sociedad" fue el nombre dado por este gobierno demócrata a un conjunto de ambiciosas políticas de inversión social destinadas a combatir la pobreza y otros problemas sociales. En el terreno 
educativo la contribución más importante a la "Gran Sociedad" fue la Ley de Educación Básica y Secundaria (Elementary and Secondary Education Act), que en su Título I establecía que se dedicarían fondos extra a centros con altas concentraciones de pobreza entre su alumnado. Con esta medida se pretendía mejorar la igualdad de oportunidades para los estudiantes pobres, si bien hasta entonces esta igualdad no había sido medida de manera sistemática en términos de resultados (Wong \& Nicotera, 2009, p. 128).

Estos dos elementos contextuales (por un lado, el clima social frente a la segregación racial en un momento en que el movimiento por los derechos civiles de la población negra había adquirido su mayor notoriedad pública y, por otro, un gobierno federal sensible a las reivindicaciones de este movimiento y activo en el intento de reducir las desigualdades) marcan, pues, el encargo legislativo al que se vincula la investigación dirigida por Coleman. Pero además, ambos elementos marcan también las expectativas hacia los resultados del propio estudio. En este sentido, la creencia general era que los resultados justificarían la lógica que había detrás de la mencionada inversión educativa de la Gran Sociedad, ya que se esperaban encontrar grandes desigualdades en los recursos de los centros a los que asistía mayoritariamente el alumnado blanco y el negro, así como que tales desigualdades explicasen las diferencias raciales en el rendimiento académico (Heckman \& Neal, 1996; Kahlenberg, 2001). El propio texto de la sección 402 de la Civil Rights Act así lo hace explícito: en él puede leerse que se encomienda un estudio sobre la falta de disponibilidad de igualdad de oportunidades educativas ("the lack of availability of equal educational opportunities"), formulación alejada de la neutralidad.

\section{Objetivos, Metodología y Contenido del Estudio}

En respuesta al citado encargo realizado por el Congreso, Coleman y su equipo diseñaron una encuesta con el fin concreto de medir el grado y las causas de desigualdad de oportunidades educativas entre los que entonces eran los principales grupos raciales y étnicos del país: afroamericanos, puertorriqueños, indios nativos americanos, mexicanos, asiáticos y blancos. La muestra incluyó en torno a 570.000 estudiantes y 60.000 docentes en unas 4.000 escuelas de todo el país, lo que por entonces la situaba como la segunda investigación de mayor tamaño en ciencias sociales (Mosteller \& 


\section{2 Álvarez-Sotomayor \& Martínez-Cousinou-Informe Coleman}

Moynihan, 1972). Aun a día de hoy sigue impresionando el tamaño muestral.

También es extenso el documento generado a partir de esta encuesta, el EEOR (Coleman et al., 1966). Su contenido se divide en dos volúmenes. El primero, de 737 páginas, es el informe en sí mismo; el segundo, de 548, recoge simplemente tablas con estadísticos descriptivos. El primer volumen se divide en 9 capítulos o secciones. Una de ellas (sección 1) es un resumen de todo el informe y otra (sección 9) constituye un extenso anexo metodológico. Es en las siete secciones restantes (de la 2 a la 8) donde encontramos los análisis referidos a la cuestión de las oportunidades educativas.

El contenido comprendido entre las secciones 4 y 8 ha pasado muy inadvertido, por más que se traten cuestiones de indudable interés para el análisis de las desigualdades de oportunidades en el contexto estudiado. Así, en la sección 4 se describen los resultados de una encuesta realizada a estudiantes blancos y negros que estaban formándose para ser maestros. Puesto que se conocía que prácticamente todo el alumnado blanco de etapas educativas inferiores era enseñado por profesores de su misma raza, mientras que a la mayoría del alumnado afroamericano le enseñaba docentes negros, analizar las posibles desigualdades en la formación de los futuros maestros de ambas razas era un factor a tener en cuenta en la desigualdad racial de oportunidades educativas. La sección 5 analiza la distribución racial y étnica del alumnado universitario en función de diversas características de los colleges en los que estudiaban. En la sexta se abordan por medio de otras fuentes las desigualdades raciales y étnicas en cuanto a escolarización y abandono escolar. La sección 7 recoge resultados de estudios de caso locales desarrollados en el marco de la misma investigación. Con ellos se pretendían ilustrar diversas problemáticas a la que se estaban enfrentando muchos centros como consecuencia del proceso de ajuste hacia un sistema escolar desagregado. Y en la sección 8 se examinan cuestiones como el efecto que tiene hablar una lengua distinta al inglés en casa sobre las habilidades lingüísticas del/la estudiante, entre otras.

Pero como comentábamos, pese a su interés, estos contenidos del EEOR han sido obviados frente a la repercusión adquirida por los resultados expuestos en las secciones 2 y 3 , que son los que, comprensiblemente, han concentrado la práctica totalidad de la atención a lo largo de este tiempo. En 
gran parte debido a que son los que derivan implicaciones de mayor calado en materia de política educativa y social.

La sección 2 está dedicada a comparar estadísticamente las escuelas a las que asistían cada uno de los grupos raciales y étnicos anteriormente mencionados. La comparación se establece en términos de un amplio abanico de características o recursos de los que disponen los centros (inputs): instalaciones, número de libros en la biblioteca, formación académica de los profesores y directores, salario del profesorado, determinados aspectos del conjunto del alumnado, etc. Por entonces, esta era la manera más extendida de entender y de medir la calidad de las escuelas (Coleman, 1969), así como la igualdad de oportunidades educativas (Coleman, 1990). Los resultados fueron sorprendentes. Aunque no siempre ocurría, es cierto que, tal y como se esperaba, se probó que los blancos asistían en mayor medida a escuelas con más y/o mejores recursos que el alumnado de minorías raciales y étnicas de la misma zona geográfica. Sin embargo -y he aquí lo sorprendente-, estas desigualdades inter-escolares resultaron ser muy estrechas. Las diferencias más pronunciadas se establecían respecto a la composición (socioeconómica, étnica y racial, fundamentalmente) del alumnado y respecto a determinadas características del profesorado que enseñaba a blancos y a negros, pero en relación al resto de variables consideradas las diferencias eran pequeñas.

Por lo tanto, los resultados evidenciaban una fuerte segregación racial, étnica y socioeconómica en las escuelas del país, pero, en contra de lo que cabía esperar, dicha segregación no implicaba fuertes desigualdades en términos de la mayoría de recursos de los que disponían las escuelas.

En cuanto a la sección 3, en ella se analizan dos cuestiones: el rendimiento del alumnado (medido mediante pruebas estandarizadas tanto de aprendizaje como de aptitud) y las relaciones entre las características de las escuelas estudiadas en la sección 2 y dicho rendimiento. Respecto a la primera cuestión, se detectaron importantes desigualdades entre los grupos raciales y étnicos estudiados. Los blancos y asiáticos presentaban un rendimiento similar en las pruebas, mientras que el resto de grupos puntuaba significativamente por debajo de estos, siendo los afroamericanos y los puertorriqueños los que se encontraban a mayor distancia.

Pero, sin duda, los resultados de mayor repercusión de esta sección y del conjunto del informe son los que se derivan de los análisis de la segunda de 


\section{4 Álvarez-Sotomayor \& Martínez-Cousinou-Informe Coleman}

las cuestiones apuntadas. Especialmente los que giran en torno a: (1) la gran importancia del origen social para el rendimiento, algo que, como reconocía el propio Coleman (1969), ya habían encontrado estudios previos, pero que quizás adquiría una mayor dimensión habida cuenta del gran tamaño muestral de esta investigación; y (2) el poco peso que tenían los factores escolares en la explicación de las desigualdades halladas (explicaban conjuntamente menos del $10 \%$ de la varianza). Respecto a la influencia del origen social sobre el rendimiento se halló, además, que esta no decrecía con los años en la escuela, lo que decía poco en favor de la esperable acción de nivelación social de esta institución. Respecto a la influencia de los factores escolares se encontró también que, dentro de su bajo poder explicativo, las características composicionales del alumnado de los centros (las socioeconómicas sobre todo) era el condicionante que más importaba, lo cual daba pie a considerar la relativa importancia del efecto de los pares. Le seguían determinadas características del profesorado. El efecto del resto de factores escolares considerados era mínimo.

Estos resultados abrieron un importante debate social sobre política educativa, algo entendible si se tiene en cuenta tanto el hecho de que el informe procedía de una ley de enorme relevancia (Alexander \& Morgan, 2016) como, sobre todo, el contexto en el que este se originó. Un contexto marcado por los rasgos descritos en el apartado anterior, pero también por las expectativas generadas hacia las reformas comprehensivas aplicadas en años previos, de las cuales se esperaba, entre otras cosas, que sirviesen como mecanismo de nivelación social.

Principalmente, el debate giraba alrededor de la que se convirtió en la interpretación más extendida de los resultados del EEOR: "las escuelas no importan, lo que importan son las familias". Malinterpretación, en realidad, si se tiene en cuenta que tal tipo de afirmación no está contenida en el informe y su simplificación extrema. De cualquier modo, esto dio pie a que se cuestionara el sentido que tenía invertir en las escuelas, con lo que se entiende que, en el contexto estadounidense, los resultados del EEOR resultaran decepcionantes para quienes esperaban poder utilizarlos para legitimar las políticas educativas de la Gran Sociedad de Johnson (Fernández Enguita, 2016, p. 39).

Por su parte, el hallazgo sobre la importancia relativa de la composición socioeconómica de los centros fue empleado para promover medidas que 
buscaran incrementar la integración socioeconómica y racial en las escuelas (Gamoran \& Long, 2007). Entre ellas, la conocida como busing, que consistía en transportar estudiantes procedentes de barrios socioeconómicamente más deprimidos a centros situados en barrios más favorecidos, tratando de combatir así el efecto que la segregación residencial tenía sobre la distribución del alumnado en las escuelas. Es sabido que el mismo Coleman apoyó inicialmente esta medida, si bien años más tarde rectificó por considerar que había generado una perversa consecuencia no deseada: la huida de muchos padres y alumnos de raza blanca hacia zonas y centros no afectados por el busing (Coleman, Kelly, \& Moore, 1975). En la misma línea, los resultados del EEOR también fueron utilizados como evidencia científica en casos judiciales sobre desegragación escolar (Wong \& Nicotera, 2009, p. 131).

El debate, por supuesto, también alcanzó fuertemente el ámbito académico. A continuación nos aproximamos a medir el impacto del EEOR en dicho ámbito. Con ello recordaremos también algunas de las principales críticas que recibió el estudio a raíz, sobre todo, del debate suscitado en torno a los últimos resultados comentados.

\section{Impacto y Debate Académico en torno al Informe Coleman}

Una primera forma (cuantitativa en este caso) de estimar el impacto académico del EEOR consiste en realizar un análisis bibliométrico del mismo. En el cuarenta aniversario desde su publicación Gamoran y Long (2007) ya realizaron un análisis de este tipo teniendo en cuenta el número de artículos publicados en revistas científicas que citaban el informe. Tomaron como fuentes el Social Sciences Citation Index, el Science Citation Index y el Arts and Humanities Citation Index. El resultado fue que el EEOR había sido citado en más de 2.700 artículos.

Para actualizar tal dato y conocer el impacto del EEOR hasta nuestros días se ha realizado un análisis bibliométrico a través de la opción "búsqueda de referencia citada" de la Web of Science (WOS), la cual permite el acceso a cinco bases de datos distintas ${ }^{3}$ además de a la colección principal de la WOS $^{4}$. La búsqueda se ha refinado únicamente por tipo de documento, de manera que solo se han contabilizado los artículos académicos en los que se cita el EEOR, excluyendo, por tanto, las citas que aparecen en reviews, 
libros o abstracts, entre otros. Siguiendo tales pasos, el número total de artículos en los que se cita el informe Coleman desde su publicación hasta $2016^{5}$ alcanza la cifra de 3.454 .

La figura 1 muestra la distribución de tales citas a lo largo del periodo considerado. En ella se observan tres etapas diferenciadas en función del impacto académico del EEOR. Una primera etapa, que iría desde el año siguiente a su publicación hasta aproximadamente 1977, en la que el Informe Coleman experimenta un impacto creciente y muy notable, llegando a alcanzar en determinados años más de 125 citas anuales. Una segunda etapa, que iría desde 1978 hasta 1997, en la que se observa una tendencia general decreciente, aunque en ningún año el informe pasa a ser ignorado por la comunidad científica. De hecho, a lo largo de esta etapa, el año en el que menos menciones al EEOR se contabilizan es 1992 con 33 citas, una cifra nada desdeñable en términos de impacto académico. Por último, una tercera etapa, que iría desde finales de los noventa hasta la actualidad, en la cual se observa de nuevo un impacto creciente del EEOR, aunque con altibajos, alcanzando puntualmente (en 2010, 2013 y 2015) más de 100 citas anuales. 


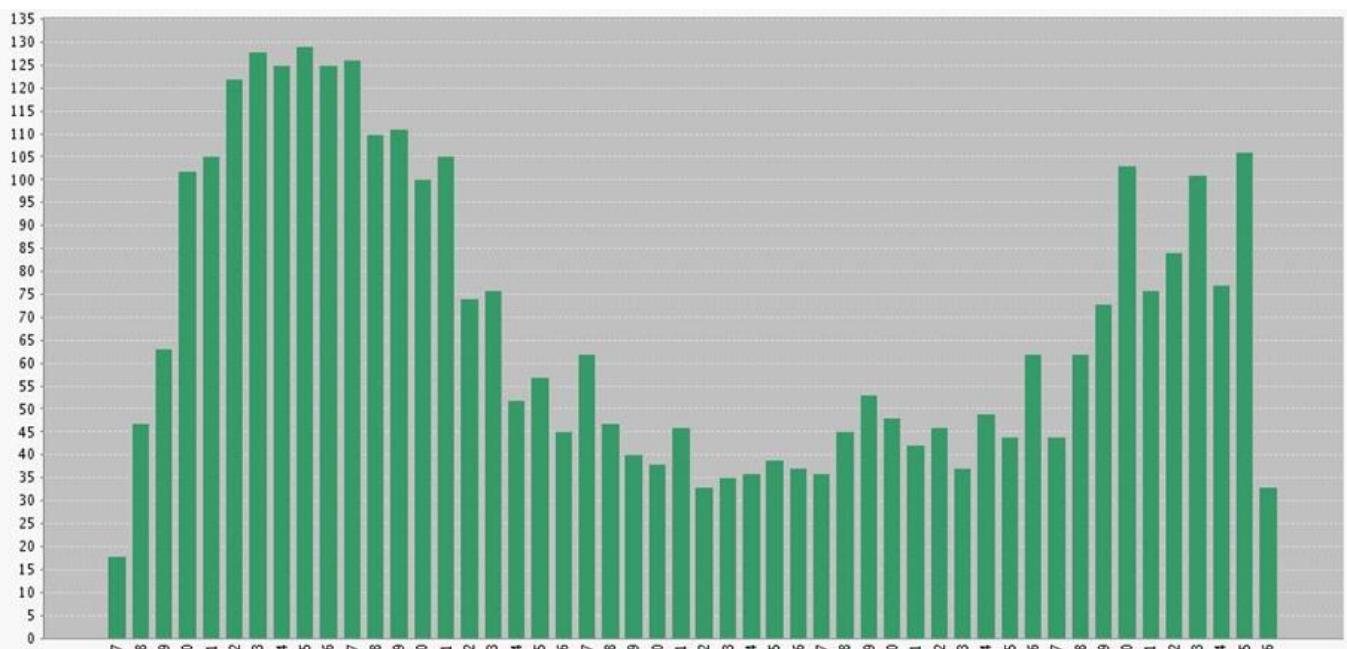

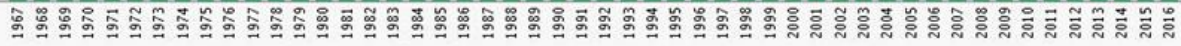

Figura 1. Evolución del número de citas del Informe Coleman (enero 1967- mayo 2016).

Fuente: Web of Science (2016).

Seguidamente, para conocer la repercusión actual del Informe Coleman en relación con el impacto de los artículos más citados del área de ciencias sociales, se ha utilizado la opción Essential Science Indicators de la WOS. En primer lugar, se ha tomado como referencia el umbral mínimo de citas anuales recibidas por el $1 \%$ de los artículos más referenciados en tal área de estudios en los últimos 10 años (Highly cited threshold). Los umbrales anuales se han comparado con las citas anuales recibidas por el EEOR a lo largo de la última década. Según se muestra en la figura 2, las citas recibidas por el Informe Coleman desde 2009 hasta 2015 le sitúan al nivel de los toppapers de la WOS (que constituyen el $1 \%$ de los mismos) dentro del área de ciencias sociales.

En segundo lugar, y para terminar de confirmar cuantitativamente el elevado impacto del EEOR dentro de las ciencias sociales, se ha tomado como referencia la media anual de citas recibida por los artículos de esta área dentro de la WOS (Citation Rates) y se ha comparado con las citas anuales recibidas por el Informe Coleman en los últimos 10 años. Tal como se muestra también en la figura 2 , mientras que en el periodo considerado un 
artículo en ciencias sociales nunca recibe, de media, más de 20 citas al año (con valores medios que oscilan entre el 16,1 de 2005 y el 0,29 de 2015), el EEOR supera con creces tales cifras, con citas anuales que se sitúan entre las 43 de 2005 y las 106 de 2015.

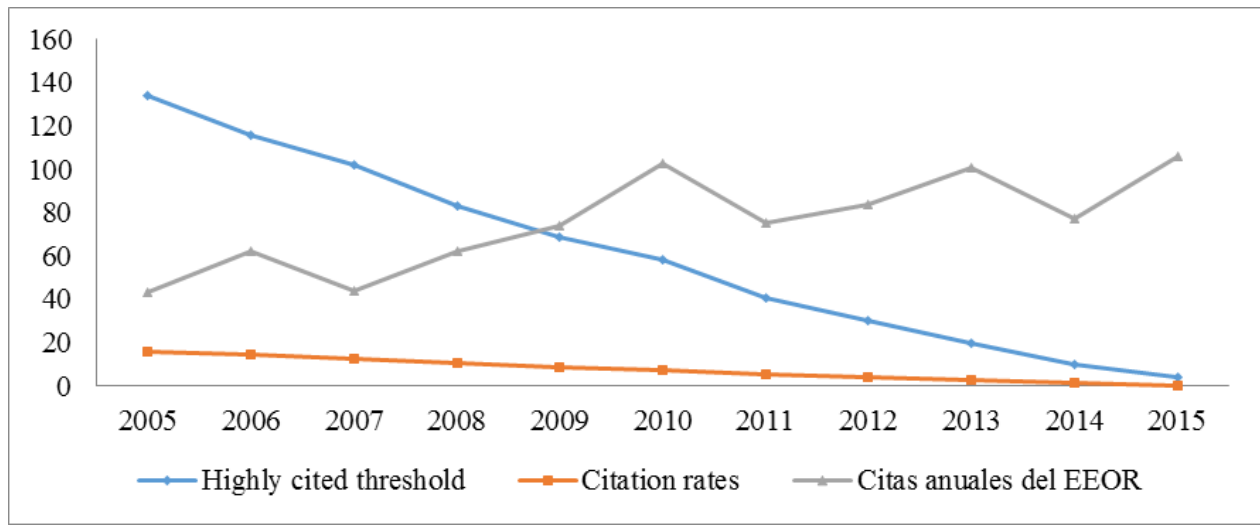

Figura 2. Impacto del Informe Coleman en relación con los top-papers y con el impacto medio anual de los artículos de ciencias sociales de la WOS (2005-2015) Fuente: Web of Science (2016).

Cabe decir que, mientras que el número mínimo de citas de los artículos más referenciados de ciencias sociales (Highly cited threshold) y la media de citas anuales de esta área (Citation Rates) han experimentado una tendencia decreciente (es decir, tanto los top-papers como la media de artículos de la WOS cada vez reciben menos citas en comparación con años anteriores), el EEOR ha experimentado un repunte en los últimos años en cuanto a referencias se refiere, situándose muy por encima del umbral mínimo anual establecido en ciencias sociales y del impacto medio anual en esta área del conocimiento.

En definitiva, estas distintas formas de medir bibliométricamente la repercusión académica del EEOR confirman el notable impacto de esta publicación. Impacto que adquiere dimensiones mayores si se tienen en cuenta dos cuestiones: primero, que no se trata en sí de un artículo académico sino de un informe elaborado para la administración pública estadounidense y, segundo, que fue publicado hace cincuenta años y por ello cabría esperar que su impacto académico hubiera descendido con el 
trascurso del tiempo.

Pero además de cuantitativamente, la repercusión académica del EEOR puede analizarse en términos cualitativos. Para ello se recuerda a continuación su protagonismo - central en algunos casos- en debates y orientaciones teóricas y metodológicas dentro de la academia. Bien sea porque se tomara como (a) referente y modelo a seguir, o (b) trabajo ante el que reaccionar críticamente. Sin ánimo de ser exhaustivos, en lo que sigue se revisan ambos tipos de reacciones.

En el primer tipo hay que mencionar cómo el EEOR fue pionero en la idea de contrastar la igualdad de oportunidades educativas a través del análisis de resultados (outputs) obtenidos por los alumnos y no de los recursos o condiciones (inputs) disponibles para estos (Gamoran \& Long, 2007). Metodológicamente contribuyó, así, a extender en la investigación educativa la utilización de estos modelos economicistas de funciónproducción. Igualmente, el EEOR popularizó el uso de muchos de los indicadores y variables que empleó para medir los inputs y outputs escolares. En estos y otros aspectos la investigación de Coleman y sus colaboradores puede considerarse antecedente de estudios nacionales de la relevancia del Inequality, de Christopher Jenks y sus colaboradores (1972), o de los internacionales y más recientes TIMSS, PIRLS y PISA (Carabaña, 2015), entre otros.

El informe contribuyó también a ampliar las miras respecto al contexto social de influencia para el rendimiento académico del individuo (Alexander \& Morgan, 2016). En una época en la que reformar la educación significaba reformar la escuela, Coleman y su equipo diseñaron un estudio que prestaba atención no solo a factores propios del entorno escolar, sino también a aquellos que tenían que ver con recursos disponibles en el entorno familiar y en el barrio o comunidad. Hoy en día este tipo de planteamiento forma parte de la rutina de la investigación educativa, pero ese no era el caso en la década de los sesenta.

Asimismo, puede afirmarse que los resultados relativos a la influencia del origen social sobre el rendimiento a los que llegaron el Informe Coleman y otros estudios de la época, dieron parcialmente sostén empírico a diversas teorías de gran calado dentro de la sociología de la educación (las de la reproducción y de la correspondencia, entre otras) que trataban de explicar el porqué de esta relación. 


\section{0 Álvarez-Sotomayor \& Martínez-Cousinou-Informe Coleman}

Por último, el EEOR es un referente clásico para los estudios cuantitativos dedicados a analizar las desigualdades educativas entre grupos étnicos y raciales.

En cuanto a las reacciones enfrentadas, a mediados de los setenta estudios macrosociológicos como el EEOR, el Inequality y otros de metodología similar comenzaron a ser criticados principalmente por: (1) no ser capaces de explicar el total de la varianza en el rendimiento académico (Karabel \& Halsey, 1977) y (2) no analizar los procesos que producían la estratificación educativa que detectaban (Bidwell, 1988). En este sentido, se criticaba que estas investigaciones trataran la escuela como una "caja negra" - se examinaba qué entraba (inputs) y qué salía (outputs) de ella, pero no qué sucedía en su interior-. Estas críticas abrieron la puerta a alternativas que nacieron, por tanto, estimuladas por las limitaciones que se detectaban en estudios como el EEOR.

Una de esas alternativas fueron los trabajos y corrientes que se propusieron comprender las desigualdades educativas desde un enfoque interpretativista (Mehan, 1991). Este tipo de trabajos, que tuvieron en la llamada nueva sociología de la educación británica y en los estudios interpretativos de los EE.UU sus principales corrientes, reaccionaron al positivismo del EEOR y estudios similares dedicándose a desentramar los procesos educativos que ocurrían dentro de las escuelas a partir de estudios microsociológicos de corte etnográfico.

Otras de estas alternativas la constituyó el "movimiento de escuelas eficaces", que nació en la segunda mitad de los setenta. El EEOR jugó también un papel decisivo en el desarrollo del mismo, ya que en parte se originó como reacción a su resultado de la escasa incidencia de los factores escolares (Báez de la Fe, 1994). Desde esta otra corriente se buscaban evidencias de lo contrario y se criticaban del estudio de Coleman algunas de las cuestiones metodológicas ya señaladas.

Finalmente, cabe mencionar un tercer tipo de crítica de calado al EEOR. Aunque, a diferencia de las anteriores, ésta ha tenido un impacto mínimo como estímulo para nuevos trabajos, de estar en lo cierto sus implicaciones serían de gran trascendencia, de ahí que resulte pertinente recogerla en estas páginas. Nos referimos a la crítica que entiende como un gran error emplear pruebas de aptitud (y no pruebas de aprendizaje) para evaluar un sistema educativo. Según sus defensores, esta decisión metodológica —adoptada en 
el EEOR y seguida por muchos de los mencionados estudios en los que influyó - sobredimensiona los efectos del origen social sobre el rendimiento y produce resultados no válidos ni fiables respecto a la estimación del impacto de los factores escolares. ${ }^{6}$

Por lo tanto, a través de este análisis del impacto del Informe Coleman puede comprobarse cómo, en un sentido o en otro, este ha servido de estímulo a multitud de investigaciones educativas durante décadas (Gamoran \& Long, 2007). Y ello tanto por cuestiones de carácter metodológico, como, sobre todo, debido al debate generado alrededor de algunos de sus resultados; especialmente los relativos a los efectos de las escuelas y del origen socioeconómico sobre las desigualdades en el rendimiento académico.

\section{Presentación del Monográfico}

Como ya se ha comentado, este número monográfico es un ejemplo más de la longeva repercusión del EEOR. Para los más críticos con él entendemos que se trata de una longevidad no deseable, en la medida en que buena parte de la misma implica que este sigue tomándose como modelo o referente. Por el contrario, para sus defensores será vista probablemente como prueba de sus virtudes y de la resistencia de sus resultados ante el paso del tiempo.

Lo que parece indudable es que, sea del modo que sea, este longevo impacto es señal también de que algunos de los debates abiertos hace ya cinco decenios por el EEOR no se han cerrado en su totalidad. El objetivo principal de este monográfico era precisamente ese: aprovechar el cincuenta aniversario de tan significada y controvertida publicación para volver a poner sobre la mesa algunos de los debates generados a partir de los resultados del Informe Coleman. Se le presta especial atención al más destacado de ellos: el que gira en torno al peso que tienen sobre las desigualdades educativas los factores escolares frente a los factores del entorno familiar. Para ello, más allá de este artículo introductorio, este número monográfico se compone de tres trabajos que se sitúan en posiciones enfrentadas respecto a dicho debate.

De un lado, el artículo de Ildefonso Marqués Perales pone en valor el EEOR destacando algunas de sus virtudes. El autor presenta en primer lugar una contextualización del informe. Tal contextualización es complementaria 


\section{2 Álvarez-Sotomayor \& Martínez-Cousinou-Informe Coleman}

a la realizada previamente en este artículo introductorio. Si aquí se ha descrito el contexto social y político estadounidense que dio lugar a la ley de la que se deriva el estudio de Coleman, Marqués nos recuerda cómo la publicación del EEOR tiene lugar después de años desde la implementación de reformas comprehensivas y otras medidas a favor de la expansión educativa en los sistemas de enseñanza de las principales economías internacionales. En dicho marco educativo se sitúa el Informe Coleman. Uno de sus principales resultados (la gran influencia del origen social sobre los resultados académicos) ponía en entredicho la eficacia de esas reformas como medio para la equilibrar las oportunidades educativas y socioeconómicas. Ello le sirve a Marqués para lanzar una de las ideas centrales de su trabajo: la dificultad de conseguir la igualdad de oportunidades (educativas en este caso) en el seno de sociedades que son profundamente desiguales. Los nuevos resultados que aporta en este trabajo a partir de la explotación de tres grandes muestras españolas continúan evidenciando la persistencia de las desigualdades educativas por razón de clase social, con lo que redundarían en esta idea.

De otro lado, en un posicionamiento muy crítico tanto hacia el Informe Coleman como hacia otros estudios y teorías a los que los autores identifican conjuntamente como integrantes del "modelo reproduccionista", se sitúan los trabajos de Ramón Flecha y Nataly Buslon, y de Jelen Amador López. En ambos se interpreta que el EEOR y estos otros estudios tienen un impacto negativo sobre la igualdad de oportunidades educativas. Asimismo, ambos trabajos utilizan experiencias y resultados de las denominadas "actuaciones educativas de éxito" (AEE) - desarrolladas en el marco de del proyecto de investigación INCLUD-ED - como ejemplo de cómo desde la escuela se pueden implementar medidas que mejoran el rendimiento académico superando el condicionamiento del nivel socioeconómico y de factores ligados al origen étnico del alumnado.

Más concretamente, el trabajo de Flecha y Buslon realiza en primer lugar un repaso por algunos de los principales trabajos, autores y teorías que influirían o formarían parte de ese "modelo reproduccionista". Recogen también algunas críticas realizadas a esos trabajos y señalan cómo algunos de sus autores modificaron sus posiciones con el tiempo. Asimismo, en contraste a esos trabajos, recuerdan las obras de Paulo Freire y Francesc Ferrer i Guàrdia, dos pedagogos que conciben la educación desde una 
perspectiva transformadora. Ambos son referentes teóricos de los modelos de AEE, los cuales son puestos como evidencia reciente de la capacidad transformadora de las escuelas.

En la misma línea si sitúa el artículo de Jelen Amador López. Amador toma como referencia el artículo A Black response to Christopher Jencks's Inequality and certain other issues (Edmonds et al., 1973). En dicho trabajo un grupo de académicos afroamericanos analizaba críticamente el Inequality (Jencks et al., 1972), el propio EEOR y otros estudios que entendían que se situaban en una línea similar a estos dos y que, desde su punto de vista, contribuían a revertir las reformas educativas y sociales que se venían realizando en materia de igualdad en los EE.UU. del momento. Partiendo de este referente, Amador plantea su trabajo como una Roma response (una respuesta gitana) frente a los planteamientos reproduccionistas que sostendrían a través de sus resultados y conclusiones la incapacidad de la escuela para superar las desigualdades socioeducativas. El artículo describe, por medio de distintos indicadores, la situación de desventaja económica, social y, especialmente, educativa que vive el pueblo gitano. Esto revelaría la necesidad de investigaciones que tengan un impacto social sobre esta comunidad. Como ejemplo de una de ellas, la autora da a conocer el caso de la escuela "La Paz", un centro con una gran presencia de alumnado gitano en el que un conjunto de actuaciones llevadas a cabo en los últimos años habrían conseguido la mejora académica de su alumnado.

\section{Notas}

1. El término afro-americano no comenzó a popularizarse en Estados Unidos hasta la década de los ochenta.

2. En concreto, la crisis se inició cuando la Guardia Nacional de Arkansas, por orden del Gobernador, impidió a nueve estudiantes afroamericanos asistir a clase en un instituto segregado de alumnado blanco.

3. La propia WOS recomienda que el análisis del impacto de un determinado documento académico (por ejemplo, el EEOR) se haga a través de la opción "búsqueda de referencia citada", en la cual se incluyen, además de la colección principal de la WOS los siguientes índices: BIOSIS Citation Index; Chinese Science Citation Database; Russian Science Citation Index; SciELO Citation Index.

4. En la colección principal de la WOS se incluyen los siguientes índices: Science Citation Index Expanded; Social Sciences Citation Index; Arts \& Humanities Citation Index; Conference Proceedings Citation Index - Science; Conference Proceedings Citation Index; Social Sciences \& Humanities; Book Citation Index-Science; Book Citation Index-Social 


\section{4 Álvarez-Sotomayor \& Martínez-Cousinou-Informe Coleman}

Sciences \& Humanities. Todos ellos se han incluido en la búsqueda para no descartar a priori ninguna referencia académica al EEOR.

5. El análisis bibliométrico se realizó en mayo de 2016, por lo que los datos correspondientes a este año no están completos.

6. Para profundizar en los argumentos de esta crítica al EEOR y a otros trabajos metodológicamente similares, consultar Carabaña (2016).

\section{Referencias}

Alexander, K., \& Morgan, S. L. (2016). The Coleman Report at Fifty: Its Legacy and Implications for Future Research on Equality of Opportunity. Recuperado de http://socweb.soc.jhu.edu/faculty/morgan/papers/Alexander_and_Mo rgan_2016.pdf

Báez De La Fe, B.F. (1994). El movimiento de escuelas eficaces; implicaciones para la innovación educativa. Revista Iberoamericana de Educación, 4, 93-116.

Bidwell, C. (1988). Willard Waller and the Sociology of Education. En D.

Willower \& W. Lowe (Eds.), Willard Waller on education and schools: A critical appraisal (pp. 39-51). Berkeley: McCutchan.

Carabaña, J. (2015). La inutilidad de PISA para las escuelas. Madrid: Catarata.

Carabaña, J. (2016). El Informe Coleman, 50 años después. Revista de la Asociación de Sociología de la Educación, 9(1), 9-21.

Civil Rights Act of 1964, Pub.L. 88-352, 78 Stat. 241 (1964).

Coleman J.S. (1969). A brief summary of the Coleman Report. En Equal Educational Opportunity (pp. 253-61). Cambridge, MA: Harvard Univ. Press.

Coleman, J.S. (1990). Equality and achievement in education. Boulder, CO: Westview Press.

Coleman, J.S.; Campbell, E. Q.; Hobson, C.J.; McPartland, J., Mood, A. M., Weinfeld, F. D., York, R. (1966). Equality of educational opportunity. Washington, DC: US Department of Health, Education $\&$ Welfare, Office of Education.

Coleman, J. S., Kelly, S. D., \& Moore, J. A. (1975). Trends in school segregation, 1968-73 . Washington, DC: The Urban Institute. 
Edmonds, R., Billingsley, A., Comer, J., Dyer, J., Hall, W., Hill, R.,

McGehee, N., Reddick, L., Taylor, H. \& Wright, S. (1973). A Black

response to Christopher Jencks's Inequality and certain other issues.

Harvard Educational Review, 43(1), 76-91.

doi:10.17763/haer.43.1.g8n8710253744kp3

Fernández Enguita, M. (2016). El Informe Coleman: Una lección de sociología y de política. Revista de la Asociación de Sociología de la Educación, 9(1), 37-45.

Gamoran, A., \& Long, D. A. (2007). Equality of Educational Opportunity A 40 Year Retrospective. En R. Teese, S. Lamb \& M. Duru-Bellat (Eds.), International Studies in Educational Inequality, Theory and Policy (pp. 23-47). New York: Springer.

Heckman, J., \& Neal, D. (1996). Coleman's contribution to education:

Theory and research styles and empirical research. En J. Clark (Ed.),

James S. Coleman (pp. 81-102). Philadelphia: Falmer.

Jencks, C., Smith, M., Ackland, H., Bane, M.J., Cohen, D., Gintis, H., Heyns, B., \& Michelson, S. (1972). Inequality: A Reassessment of the Effects of Family and Schooling in America. New York: Basic Books.

Kahlenberg, R. D. (2001). Learning from James Coleman. Public Interest, $144,54-72$.

Mehan, H. (1992). Understanding inequality in schools: The contribution of interpretive studies. Sociology of education, 65(1), 1-20. doi: $10.2307 / 2112689$

Karabel, J., \& Halsey, A. H. (Eds.). (1977). Power and ideology in education. New York: Oxford University Press.

Kilgore, Sally B. (2016). The Life and Times of James S. Coleman. Education Next, 16(2), 8-16. Recuperado de http://educationnext.org/life-times-james-s-coleman-school-policyresearch/

Mosteller, F. \& Moynihan, D. A. (1972). Path Breaking Report: Further Studies of the Coleman Report. En F. Mosteller \& D. A. Moynihan (eds.), On Equality of Educational Opportunity, Papers Deriving from the Harvard University Faculty Seminar on the Coleman Report (pp. 3-69). New York: Vintage Books. 
Wong, K.K., \& Nicotera, A.C. (2004). Brown v. Board of Education and the Coleman Report: Social science research and the debate on educational equality. Peabody Journal of Education, 79(2), 122-135. doi: 10.1207/s15327930pje7902_8
Alberto Álvarez de Sotomayor is Professor at the Department of Social Sciences and Humanities at University of Córdoba.
Gloria Martínez Cousinou is Professor at the Department of Psichology, Sociology and Social Work at the Loyola Andalucía University
Contact Address: Direct correspondence to Alberto Álvarez de Sotomayor, Department of Social Sciences and Humanities, Faculty of Education, University of Córdoba, Avenida San Alberto Magno s/n. Córdoba, Spain. E-mail: aasotomayor@uco.es 